Revista da Sociedade Brasileira de Medicina Tropical

Journal of the Brazilian Society of Tropical Medicine

Vol.:52:e20190334: 2019

doi: 10.1590/0037-8682-0334-2019

\title{
Letter
}

\section{De novo histoid leprosy - Further points of focus}

\author{
Sajitha Venkatesan ${ }^{[1]}$ and Pugazhenthan Thangaraju ${ }^{[2]}$
}

[1]. Central Leprosy Teaching and Research Institute, Department of Clinical Division, Chengalpattu, India. [2]. All India Institute of Medical Sciences, Department of Pharmacology, Raipur, Chhattisgarh, India.

\section{Dear Editor:}

We have found the case report "De novo histoid leprosy in a Colombian patient with multiple skin nodules on the ears and extremities" by Lina Marcela Piedrahíta-Rojas ${ }^{1}$ an interesting read. It is known that histoid leprosy is an uncommon form of Lepromatous leprosy with distinct clinical, nodular, histopathological, and fluctuable immunological features.

The authors reported a very interesting case of histoid leprosy diagnosed in a young patient presenting with skin nodules. As the authors have correctly pointed, early diagnosis with appropriate treatment regimens are recommended for the management of histoid leprosy as they are important reservoirs of Mycobacterium leprae and cause transmission of the bacteria.

Here we have included some additional points regarding the management of histoid leprosy that might help the readers as well as the authors. Although the patient did not develop lepra reaction during treatment in the study, in our experience there is always a high risk of developing reactions in the near future as patients with histoid leprosy have an increased risk of developing both types of lepra reactions. It would also be of help to calculate the morphological index in the biopsy. This would not only help us understand the drug sensitivity status, but would also approximately predict the chances of relapse or the risks of developing lepra reaction in the future based on either the morphological index or the log of the bacteriological index. It is therefore advisable to mandatorily determine both the indices in patients with histoid leprosy. With regards to disease relapse, it is advisable to observe the young patient for a surveillance period of 5 years $^{2}$ prior to commenting on the possibility of relapse.

Corresponding Author: Dr. Pugazhenthan Thangaraju.

e-mail: drpugal23@gmail.com

Orcid: 0000-0002-4090-2004

Received 06 July 2019

Accepted 24 July 2019
Another important point that should be considered in histoid leprosy cases is screening all the family members clinically, and if feasible perform slit-skin smear testing on the family members. Currently, the World Health Organization (WHO) recommends a single dose of rifampicin (SDR) ${ }^{3}$ as chemoprophylaxis for all individuals coming in contact with the patient diagnosed with leprosy. Since bunches/abundant bacilli were observed in the biopsy in this case, it is a good and safe practice to screen and administer chemoprophylaxis to at least his close household contacts.

To conclude, there should be a high index of suspicion in the treatment of histoid leprosy, and it is essential to continue the surveillance in the concerned national program for identifying new and clinically relapsed cases, rather than to wait for self and voluntary reporting. Various strategies have been operational in India as it harbors 60 percent ${ }^{4}$ of the global leprosy cases. Two major strategies, including the prevention of transmission and leprosy detection campaigns help detect hidden leprosy cases in a community ${ }^{5}$. For preventing transmission, chemoprophylaxis with a single dose of rifampicin is recommended. Vaccine trials are also being attempted for preventing disease transmission. Leprosy case detection campaigns are in full form and are actively identifying cases in the high endemic regions and low endemic areas, thus helping in the detection of hidden leprosy cases. The Sparsh awareness program is also actively functional in India. Authors in Columbia ${ }^{6}$ have reported that the cases of leprosy are on the rise and the program should be strongly implemented for preventing a rise in the number of cases. Therefore, the aforementioned strategies could be also be implemented in a Columbian set up.

\section{REFERENCES}

1. Piedrahíta-Rojas LM, Díaz CJ, Escandón-Vargas K. De novo histoid leprosy in a Colombian patient with multiple skin nodules on the ears and extremities. Rev Soc Bras Med Trop. 2019;52:e20160502.

2. Follow up period. Guidelines for the Diagnosis, Treatment and Prevention of Leprosy. http://nlep.nic.in/pdf/ WHO $\%$ 20Guidelines\% 20 for \%20 leprosy.pdf. Retrieved on 28/02/2019. 
3. Single dose rifampicin. Guidelines for the Diagnosis, Treatment and Prevention of Leprosy. http://nlep.nic.in/pdf/ WHO $\%$ 20Guidelines\% 20for \%20 leprosy.pdf. Retrieved on 28/02/2019.

4. India contributes $60 \%$ of global leprosy cases. https://www.who.int/ neglected_diseases/news/India_massive_leprosy_case_detection campaign_reaches_320_mill/en/. Retrieved on 28/02/2019.
5. Thangaraju P, Venkatesan S, Showkath Ali MK. Final leprosy push: Out of society. Indian J Community Med. 2018;43(1):58-9.

6. Cardona-Castro, N. Leprosy in Colombia. Curr Trop Med Rep .2018;5:85. https://doi.org/10.1007/s40475-018-0145-7 